\title{
Prompt comprehension in UNIX command production
}

\author{
STEPHANIE M. DOANE \\ University of Illinois, Champaign, Illinois \\ and
}
DANIELLE S. MCNAMARA, WALTER KINTSCH, PETER G. POLSON, and DEBORAH M. CLAWSON
University of Colorado, Boulder, Colorado

\begin{abstract}
We hypothesize that a cognitive analysis based on the construction-integration theory of comprehension (Kintsch, 1988) can predict what is difficult about generating complex composite commands in the UNIX operating system. We provide empirical support for assumptions of the Doane, Kintsch, and Polson $(1989,1990)$ construction-integration model for generating complex commands in UNIX. We asked users whose UNIX experience varied to produce complex UNIX commands, and then provided help prompts whenever the commands that they produced were erroneous. The help prompts were designed to assist subjects with respect to both the knowledge and the memory processes that our UNIX modeling efforts have suggested are lacking in less expert users. It appears that experts respond to different prompts than do novices. Expert performance is helped by the presentation of abstract information, whereas novice and intermediate performance is modified by presentation of concrete information. Second, while presentation of specific prompts helps less expert subjects, they do not provide sufficient information to obtain correct performance. Our analyses suggest that information about the ordering of commands is required to help the less expert with both knowledge and memory load problems in a manner consistent with skill acquisition theories.
\end{abstract}

This research applies cognitive theory to an applied problem in human-computer interaction. Modern computerbased systems make numerous cognitive demands on users. The sheer volume of information presented is often overwhelming, and this can be confounded by obscure human-computer interface designs. Detailed analyses of interface designs in the context of cognitive theories have proven fruitful. Researchers espousing theoretical approaches to human-computer interaction recognize that there are multiple determinants to the problems that users have with complex systems, and that a theoretical approach is required if these determinants are to be disentangled (see, e.g., Card, Moran, \& Newell, 1983). Theory-based approaches seem the most profitable for providing causal analyses of the real difficulties users have with complex systems, and have the best chance for generalizing to other system interface designs. In addition, applied domains are fruitful environments for the investigation of fundamental issues in cognition, and applied problems pose unique

This research was supported by NSF Grant IRI 8722792. The authors thank Clayton Lewis for his insightful comments. We also thank Susan Dumais and three anonymous reviewers for comments on earlier versions of this manuscript. We thank R. Gary Dungca and Zhihua Long for their assistance in completing this research. Please address all correspondence to Stephanie Doane, Department of Psychology, 603 East Daniel Street, University of Illinois, Champaign, IL 61820. challenges for theories of cognition to address (see, e.g., Polson \& Lewis, 1990).

The goal of this study is to provide empirical support for the assumptions about users' knowledge of UNIX and users' memory processes that have been proposed by the Doane, Kintsch, and Polson $(1989,1990)$ UNIX comprehension construction-integration model (UNICOM) for generating complex commands in UNIX. In so doing, we specify the role of this theory in understanding user action planning, and we illustrate a general methodology that may be used to examine complex knowledge and memory process assumptions made by other cognitive models.

\section{Theoretical Background}

In UNICOM, we model the understanding of task instructions and the generation of appropriate action plans. Van Dijk and Kintsch (1983) and Kintsch (1988) make the point that comprehension of text that describes a problem to be solved (e.g., an algebra story problem) involves retrieval of relevant factual knowledge, formulation of an effective solution plan for that problem, and utilization of appropriate procedural knowledge (e.g., knowledge of algebraic and arithmetic operations). In UNICOM (Doane, Kintsch, \& Polson, 1989, 1990), this is modeled by the retrieval of both relevant general knowledge and specific knowledge about UNIX command and redirection syntax, and then formulating action plans for producing 
UNIX commands. Thus, understanding a production task means generating a mental representation of the syntax and concepts in the operating system (e.g., files, commands, special input/output, or $\mathrm{I} / \mathrm{O}$, redirection symbols) and the relationships between those items, and then using this information to generate an action plan. The difference between novice and expert users is represented in the UNICOM model by deficits in knowledge about UNLX (e.g., knowledge of pipes, and command names) and deficits in processes assumed to be required for UNIX items to be organized together into an action plan. In the present empirical study, we probed these deficits further by providing users at varied levels of expertise with textual descriptions of UNIX command goals, which were followed by help prompts.

A fundamental component of the UNIX user interface is that composite commands can be created by concatenating simple commands with the use of advanced features that redirect command input and output. The composite commands act as small programs that provide unique, user-defined functions. For example, the single command "ls" will print the filenames in the current directory on the screen. The single command "Ipr" used in combination with a filename (i.e., "lpr file") will display the contents of the file on the line printer. If a user wishes to print the filenames of the current directory on the line printer, this can be accomplished in one of two major ways.

The first method requires the use of redirection symbols that store intermediate steps in files. For example, the " $>$ " symbol redirects the output of a command to a file. Thus, the command "Is $>$ file" would output the names of the files in the current directory into "file." If this were followed by the command "Ipr file," the file names would be printed on the line printer. The second method requires fewer keystrokes, and as such, it is more streamlined. The use of pipes (" $\mid$ ") allows commands to be combined to produce the command "ls|lpr." In this example, the output from the "ls" command flows directly into the "Ipr" command. Thus, one can think of a pipe as a plumbing pipe, through which information flows directly.

We wish to determine what kinds of knowledge users need in order to use these advanced features (e.g., input/output redirection). To know the specific action (e.g., to know that the command "Is" lists file names) is critical for plan execution, but simply to know the elements is not sufficient to develop an effective action plan.

In previous empirical work, UNIX users at varied levels of expertise have been provided with textual descriptions and have been asked to produce legal UNIX commands that require the redirection of standard input and output (i.e., "composite"' commands; see Doane, Pellegrino, \& Klatzky, 1990). The findings suggest that novices and intermediates have knowledge of the elements of the system; that is, they can successfully produce the single and multiple commands that make up a composite. They cannot, however, put these elements together by using pipes and/or other redirection symbols to produce the composite commands. It is important to note that the subjects had difficulties with composites even though they were allowed to produce them by using any legal means to accomplish the specified goal (e.g., "ls > temp; lpr temp" for "ls $\mid l p r ")$. The symbols that enable $I / O$ redirection are fundamental design features of UNIX, and these features are taught in elementary computer science courses; but Doane, Pellegrino, and Klatzky (1990) have demonstrated that these features can only be used reliably after extensive experience (e.g., experts had, on the average, 5 years of experience with UNIX).

We now turn to describing a computational model that was developed to explain some of the reasons that users have such difficulties when they attempt to produce composites. Discussion of the model will precede discussion of the prompting study. An understanding of the details of the model and of our resulting knowledge analysis is requisite for appreciating the motivation for the prompt study.

\section{UNICOM Construction/Integration Model}

UNICOM (Doane, Kintsch, \& Polson, 1989, 1990) was developed to determine why the Doane, Pellegrino, and Klatzky (1990) users had such difficulties in attempting to generate composite commands. Basically, by specifying the knowledge that users require in order to produce composite commands in enough detail so that UNICOM could run, we developed detailed hypotheses about the large knowledge and memory process demands that such tasks place on the user. ${ }^{1}$ The goal of the prompting study, then, was to provide empirical support for these hypotheses. We will now describe the construction-integration model (Kintsch, 1988), but only in enough detail to allow the reader to comprehend how the model guided our analysis of the problems that users have with the UNIX system. (For general details on the general model, see Kintsch, 1988; for details on the UNIX constructionintegration model, UNICOM, see Doane, Kintsch, \& Polson, 1990.)

Overview. The construction-integration model is a cognitive architecture that is based on a general theory of discourse comprehension and that uses knowledge in the form of associations. In the context of the present research, using UNIX refers to the comprehension of brief instructions to produce legal UNIX commands and associating this instruction with knowledge about UNIX in order to develop an action plan. The focus of our analysis is not so much on understanding the text per se, but on the way in which these instructions activate the UNIX knowledge relevant to the performance of the specified task.

The model activates knowledge in parallel through activation and selection of contextually relevant knowledge. It is a situated cognition model, in that it does not plan ahead; rather, the model uses facts about the current state of the world to guide knowledge activation and selection in a step-by-step fashion. The instructional text and the 
current state of the operating system serve as cues for activation of the relevant knowledge, and for organizing this knowledge to produce an action sequence.

Classes of knowledge. The UNICOM model requires that three classes of knowledge be available to simulate command production (see Table 1). The first class of knowledge used by the model is called world knowledge. This represents the state of the world at the current moment. Examples of world knowledge include knowledge of the current task, what files exist on the current directory, what directory you are at, and what system is in use (UNIX). These facts are contextually sensitive and fluid, and many of them would change for a different task and with further developments in this task. For example, if a file is deleted, or if the state of the system changes (e.g., a user moves from the system level to the editor), the world facts will reflect this change.

The next class of knowledge, general knowledge, is similar to Anderson's declarative knowledge in ACT* (Anderson, 1983). General knowledge refers to facts about UNIX. To facilitate discussion of the UNICOM knowledge base, we will start with the command "nroff -ms ATT2 > ATT1" as an example. This command formats the contents of the file ATT2 by using the utility "nroff" and the "-ms" macro package; it then stores the formatted contents of ATT2 in the file ATT1. Four types of general knowledge are required for a user to produce a composite command. UNICOM must know two types of knowledge that are syntax dependent, and two general types of knowledge that are syntax independent. We will call syntax independent knowledge conceptual knowledge, to facilitate exposition. As shown in Table 1, the model must know: (1) command syntax, (2) I/O redirection syn$\operatorname{tax}$, (3) conceptual facts about the redirection of input and output, and (4) conceptual facts about command redirection.

Table 1

Examples of Knowledge Required by UNICOM Model

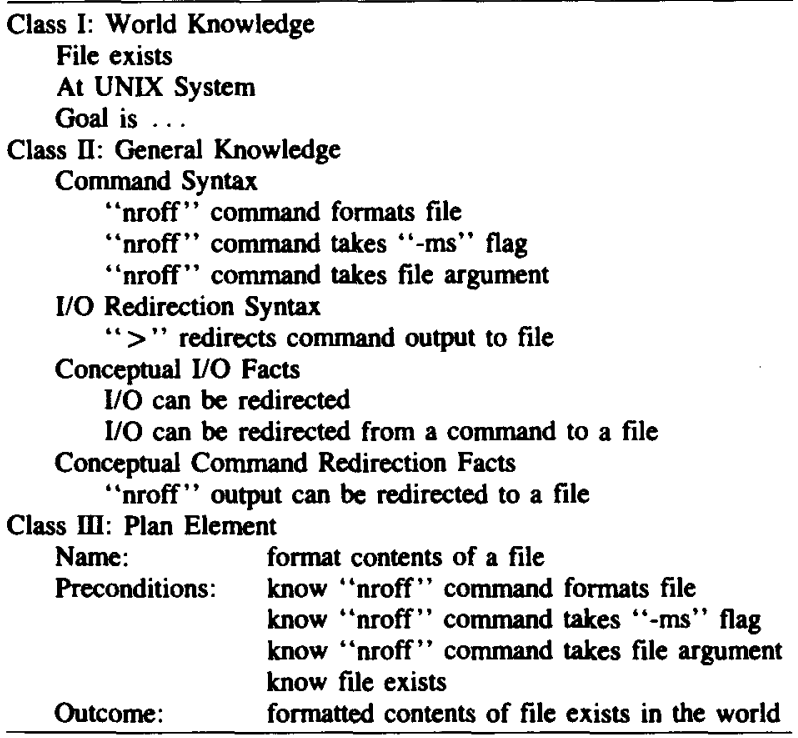

An example of command syntax knowledge would be to know the "nroff" command and the "-ms" flag. An example of $\mathrm{I} / \mathrm{O}$ redirection syntax would be to know the " > " redirection symbol. Examples of conceptual facts about the redirection of input and output include the conceptual knowledge that redirection of input and output can occur between commands. This is separate from the syntaxspecific knowledge of $\mathrm{I} / \mathrm{O}$ redirection symbols. (Some users appear to know that redirection can occur, but do not know the specific syntax.) Finally, an example of a conceptual fact about command redirection would be the knowledge that the output of "nroff" can be redirected to a file. This knowledge is necessary, because UNIX commands are not consistent in their I/O structure (e.g., an "ls" cannot take input from another command, but "sort" can).

Finally, the third class of knowledge, called plan elements, consists of the executable forms of knowledge about UNIX. They are analogous to productions in ACT* (Anderson, 1983). Plan elements describe actions that can be taken in the world, and they specify conditions under which actions can be taken. Thus, users have condition-action rules that they can consider and execute if conditions are correct. In the model, plan elements have three parts. The first is the name of the plan element. The second component contains the preconditions that must be present either in the world or in the general knowledge if the plan element is to fire. For example, if a file to be formatted does not exist in the world knowledge, then a plan element that requires that the file exists will not be able to fire. Finally, there is an outcome component of plan elements. These contain facts that will be added to the world if the plan element fires. For example, once a file is formatted, the world knowledge will change to reflect the fact that the formatted contents of the file exist in the world.

Many plan elements may be selected in sequence to form an entire action plan (described below). The model operates in a cyclical fashion. The model fires the most activated plan element whose preconditions are satisfied in the world. When it fires, the outcome of the selected plan element is added to the world. The selection of the next plan element is determined by the modified contents of the world. For plans to be selected for execution, they must be contextually relevant to the current task (as dictated by the world knowledge), and the facts that allow them to fire (preconditions) must exist in the knowledge base. For example, to execute the plan to do a "nroff" on a file, the plan element to format a file must be activated, and to be activated, it must be relevant to the current task (i.e., the command to count the number of words in a file, "wc," would not receive much activation if the user's task was to alphabetically arrange the contents of a file, because it is not contextually relevant). In addition, the preconditions of the plan element including the prerequisite state of the world and the presence of general knowledge must be fulfilled. For example, the user must be at the system level (state of the world knowledge), 
they must know the "nroff" command, and the file that is to be formatted must exist in the world (see Table 1).

UNICOM knowledge base. Now that we have defined the three classes of knowledge that the model must have available, we turn to describe the knowledge set included to simulate UNIX command production in the UNICOM construction-integration model. We will focus on the use of the model as a tool for explicating the knowledge required to produce legal UNIX commands. We will then use this knowledge to design prompts and analyze the data resulting from the main focus of this research-that resulting from the prompt study. First, we will describe the knowledge required by a model of the expert user in order to produce single commands by themselves; then, we will describe the additional knowledge and processing required to produce composite commands.

Our expert model includes knowledge necessary to produce the single commands "cat," "cmp," "edit,"

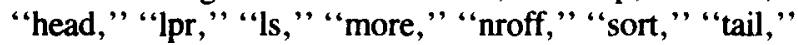
and "wc." These commands were later combined into composites in the Doane, Pellegrino, and Klatzky (1990) study. We previously discussed the example command "nroff," which formats the contents of a file, but all other single commands are similar in terms of the type of knowledge prerequisites. Recall that for a single plan element to be executed, it must be activated by being relevant to the task at hand, and the world knowledge and general knowledge that are prerequisites for the plan element to fire must exist in the knowledge base. To execute the plan element for "nroff" (i.e., the "format contents of a file" plan element in Table 1), the model must have syntax knowledge of the "nroff" command and world knowledge that the file to be formatted exists. It does not require knowledge of I/O syntax, conceptual knowledge of I/O redirection, or conceptual knowledge about command redirection. Once the "nroff" plan element is fired, the fact that the formatted contents of the specific file formatted exist will be added to the world knowledge.

Composites require the sequence-dependent coordination of commands using one or more $\mathrm{I} / \mathrm{O}$ redirection symbols (e.g., "|," " >," etc.). To accomplish this, the model requires much more knowledge than that required for single commands. In addition to knowledge that is required for singles such as certain world knowledge and knowledge about the specific commands, to produce a composite, the model must have knowledge of $\mathrm{I} / \mathrm{O}$ syntax, conceptual knowledge of $1 / 0$ redirection, and conceptual knowledge of command redirection. We discuss this in the context of the "nroff -ms ATT1>ATT2" composite command.

First, the model must have conceptual knowledge about redirection-for example, that standard input and output can be directed (see Table 1). It is interesting to note that this type of information is much more conceptual or abstract than the previous knowledge required for command completion. That is, it is not attached to a specific command or output. Chi and others have noted that novices have much more difficulty in acquiring abstract knowledge than they do in acquiring more concrete facts (e.g.,
Chi, Feltovich, \& Glaser, 1981). Thus, we would expect novice subjects to have difficulty with this type of knowledge.

Second, the model must have syntactic knowledge about redirection. For example, the model must know that a filter " $>$ " redirects output of commands to files.

Third, conceptual knowledge of the redirection properties of each command to be included in the composite is required. For example, to execute a composite that includes redirecting the output of the command "nroff,", the model must know that "nroff" output can be redirected to a file. This third type of knowledge is critical to understanding the difficulties that people have with UNIX composite production. Recall that many users can produce the single commands, know the redirection symbols, and yet not be able to put these elements together to produce a composite command. One explanation for this pattern of performance is that the sheer number of facts a user must know to produce a composite is significant, and these knowledge prerequisites cause performance degradation. Moreover, in UNIX, the structure of the redirection properties of commands is not consistent. So, for example, the redirection properties of "ls" are very different from those of "sort." Both the input and output of the "sort" command can be redirected from or to other commands. In contrast, only the output of "Is" " can be redirected. If one uses the analogy between the "shape" of the redirection properties of UNIX commands and the shape of puzzle pieces, then UNIX commands have very different shapes. There are cognitive consequences to the different shapes of the commands-users cannot assume the redirection properties of a command that they are using. These properties must be memorized and retrieved for each individual command.

Fourth, the model requires plan elements that represent the ability to produce the redirection symbols such as pipes and filers. For example, to use a pipe, it must have a "use pipe" plan element.

Finally, the model must have knowledge of the temporary state of the information flowing between commands. We will discuss this in the context of a different example that requires the use of pipes, to execute a "sort filel |tail|lpr" command in response to the instruction to print the last 10 alphabetically arranged lines of "filel" on the line printer. To process this request, the model must select the "sort" plan element, then keep in memory the result of performing the "sort" (the sorted contents of "file l" exist), then select the tail plan element, then keep in memory the result of executing the tail (the last 10 sorted lines of "file1" exist), and then select the "lpr" plan element and store the result of executing the "Ipr" (the last 10 sorted lines of "filel" exist on the line printer). The model must sequence commands properly to achieve the desired result. That is, a "tail filel | sort |lpr" would result in a very different outcome than would the correct command.

Summary of knowledge required. The expert model uses all the knowledge detailed above to complete a composite command. As another example, let us look at how 
the model executes the command "sort filel |head." The model would first activate the goal plan element, which is to take the "head" of the sorted contents of the file. But to do this, the sorted contents of the file must exist. They do not exist, and the "head" plan element cannot fire. Thus, the first plan element chosen for execution is the "sort file1" plan element. This element is fired if the model knows the syntax knowledge required for the "sort" command, if the file exists, and if the user is at the system level. Following execution of the "sort" plan element, the fact that the sorted contents of the file exist in the world is added to the knowledge base. Then the model must chain the results of "sort" to the "head" command, using the "use pipe" plan element. To do this, it must know both conceptual and syntactic I/O redirection facts. It must know that input and output can be redirected, that the pipe redirects input and output between commands, that the output of "sort" can be redirected to another command, and that the destination command, "head," can take input from another command. Once the "use pipe" plan element is fired, the trace of the outcome of using the "pipe" plan element is added to the world knowledge. Finally, the model must select the "head" command and store the knowledge that a head on the sorted contents of "file" exists in the world, and then the planning process is complete.

Note that, as previously stated, the model must keep track of many intervening results while planning a composite, and it must do so without any guidance from the system. This is what we conclude that users must do as well. We were in fact surprised by the sheer number of additional facts required for composites to be produced. In addition to greater knowledge about redirecting input and output, the expert model had to know the specific redirection properties of each command in order to keep it from making overgeneralization errors (i.e., using " ls" on the right side of a pipe). Further, the amount of facts that the model had to add to its memory of events happening in the world was also very surprising. The model has to keep track of intervening results when planning a string of single commands, but the process is much less precise than those required here. For example, the model must remember whether a command has been completed or not. But the model does not have to recall the exact output of that command as it relates to the next thing to do. In fact, once a command is done, the exact results can be dropped from memory. To execute the next command in a series of single commands, the model needs only to remember that the last command was executed. Its exact result is not used in any later commands.

Theoretical summary of UNICOM analysis. Doane, Kintsch, and Polson $(1989,1990)$ assumed that the mechanisms involved in integrating and ordering knowledge that are required for the production of a composite would result in knowledge demands and possible memory load problems. Item knowledge and ordering processes not only are involved in the composition of UNIX commands but also are important for understanding problem solv- ing and skill acquisition in general. The literature on expertise and skill acquisition (e.g., Chi et al., 1981; Larkin, 1983) suggests that less expert individuals may know the separate facts in a domain, but that they are unable to use them productively in the sense described by Wertheimer (1982). In addition, it is assumed that experts possess knowledge structures that decrease this memory load, whereas novices do not (see, e.g., Chase \& Ericsson, 1982). Understanding how individuals utilize textual instructions and experience to transform their factual knowledge into effective problem solving procedures is an important general issue in skill acquisition.

We also note that in our analysis of the knowledge and memory processes that enable individuals to chain elements together, we are working within the context of a theory of comprehension that has been used to explain algebra story problem comprehension (Kintsch, 1988), as well as the solution of simple computing tasks (Mannes $\&$ Kintsch, 1991). Furthermore, the theory of comprehension used here was originally developed in the context of story comprehension (Kintsch, 1988) and has been widely applied in the context of narration and descriptive texts. Thus, rather than developing an idiosyncratic knowledge analysis, we are performing our research in the context of a general architecture of cognition. As such, we contribute to the goal suggested by Newell (1987) to develop a "unified theory of cognition."

\section{Research Goals}

The knowledge analysis resulting from UNICOM simulations suggested that less expert users may have trouble because they are lacking in one of the four knowledge types specified above (i.e., command syntax knowledge, V/O syntax knowledge, conceptual facts about $\mathrm{I} / \mathrm{O}$ redirection, and conceptual facts about command redirection), or because they are lacking in the ability to sequence the items and keep track of the intermediate results. Again, we presume that the latter is a consequence of knowledge deficits, working memory load problems, or both. However, empirical support for these hypotheses is needed. One way to examine knowledge is to ask users to produce composite commands and then give them help prompts that systematically provide them with the four knowledge types mentioned above, or to assist them with the process of sequencing the command items and keeping track of the intermediate results. We expected that novices would be helped somewhat by prompts that provide syntax and conceptual knowledge (e.g., that tell about use of pipes or give command names), and that they would need further assistance to be able to properly sequence elements of a composite command. This assistance was given in the later prompts (see Prompt 6 and Prompt 8 in Table 2). We expected that experts would also be helped somewhat by prompts that provide syntax knowledge because they might not recall some of the command names. But we expected that they would not benefit from prompting on conceptual knowledge of V/O redirection and command redirection and that they possessed the knowledge that would allow them to keep track 
of the sequence of operations without much difficulty (e.g., Chase \& Ericsson, 1982).

Our goals in this study, then, were to determine more precisely what users at different levels of expertise know about UNIX, what information users lack when they produce erroneous commands, and what information (i.e., prompt contents) helps them. We used a prompting paradigm to assess the knowledge and processes of users with various levels of expertise. We assumed that users have different amounts of the required four types of knowledge and that displaying the prompts that help with each respective type of knowledge would affect subsequent user performance. Thus, we scored production attempts made after to each prompt presentation for amount of command syntax, I/O syntax, conceptual I/O redirection, and conceptual command redirection knowledge. If the prompts that are intended to help with the respective types of knowledge do influence performance on subsequent productions, and if this change in performance is different for novices and experts, this would support the hypothesis that these four types of knowledge are indeed required to produce UNIX composites and that they are present in different amounts in novices and experts.

We made similar assumptions about the process knowledge (i.e., keeping track of intermediate results). We expected that experts would be able to accomplish this without the benefit of prompts for the most part. In contrast, novices were expected to require assistance with ordering elements of a command and conceptual command redirection knowledge even after they had been presented with prompts that provided all of the command syntax, L/O syntax, and conceptual I/O knowledge that they would need in order to successfully produce a composite.

\section{METHOD}

\section{Subjects}

Twenty-two computer science and electrical engineering majors with 6 months to 6 years of experience with UNIX were paid \$20 for their participation. A preexperimental questionnaire was administered to determine the users' experience with UNIX and other operating systems, their history of computer training, and their selfassessment of their computer skills. All subjects had received prior instruction about redirecting standard input and output in their coursework and had experience in using redirection symbols to complete coursework or other tasks with UNIX.

Following the method outlined in Doane, Pellegrino, and Klatzky (1990), subjects were classified into expertise groups. Novices $(n=10)$ had less than 1.25 years of experience with UNIX and no operating systems courses; intermediates $(n=8)$ had 1.25 to 3.0 years of experience with UNIX, and some had operating systems courses; and experts $(n=4)$ had greater than 3 years of experience with UNIX and all had taken an operating systems course.

\section{Apparatus and Materials}

All production tasks were performed on a Macintosh II with a large-screen monitor. The experiment was controlled by a program written in SuperCard. The stimuli were task statements, a fixed directory of file names, and a series of help prompts, all of which were displayed on the screen, as well as three physical $3 \times 5$ in. error cards presented by the experimenter. The subjects responded by typing at the keyboard a command or series of commands to accomplish a given task and then used the mouse to click on a displayed button to cause the program to evaluate their answers.

The task instructions described actions that could be accomplished best by combining two or three commands through the use of redirection (i.e., composite problems). An example is the task shown in Table 2, for which the command sequence would be "nroff -ms ATT2 > ATT1." Accompanying the task statement was a fixed directory, which listed of all file names that were used in the experiment; this directory assured the subjects that any files referenced in the task statement existed already and did not need to be created. The 21 composite problems presented by these task statements were presented in one of three orders, order being counterbalanced across level of expertise. Together, the problems required the use of 10 different utilities (e.g., "Ipr," the utility that prints a file on the line printer; "cmp," the utility that compares the contents of two files; and "cat," the utility that displays the contents of files); and four different I/O redirection symbols (e.g., " $>$ ", "l," " $>>$," " > "). The answers to the composite problems are shown in Table 3.

The composite problems were chosen for two reasons. First, we are trying to understand how people develop novel action plans to produce composites. Thus, we did not wish to use familiar, even habitual composite productions (e.g., "Is $\mid$ lpr") that subjects could simply retrieve from memory. It is reasonable to assume that even though some of the composites used in the present study would not be familiar to the subjects, they could be produced if a user possessed the syntax of the language, the concepts of redirection, and the tracking of intermediate results that must occur if one is to sequence them together. ${ }^{2}$ The second reason for choosing this set of composites is that they are identical to those produced by subjects in the Doane, Pellegrino, and Klatzky (1990) study and as such have been previously tested. When both the commands and the instructions to produce the commands were created, they were reviewed by both high-level experts (e.g., system administrators) and intermediate users for their legality and for their comprehensibility. The resulting 21 commands passed the review without difficulty.

For incorrect responses, a series of help prompts was designed to address specific deficits in the subjects' UNIX knowledge. These prompts were displayed on the screen one at a time in a fixed order, regardless of the type of error that the subject had made. That is, the system had a set of prompts for each problem, and these were displayed in turn when the subject made an error, regardless of the nature of the error. The system simply parsed an answer to determine whether it contained the required syntax in the requisite order, and if it did not, the system would display the next prompt in the sequence. The help prompts were developed to address the most probable causes of error as suggested by the UNICOM model of UNIX command production.

There are seven different areas in which the help prompts could assist the subjects, and these are best described by referring to the example in Table 2. Prompt 1 parses the task statement into relevant command concepts. Prompt 2 identifies actual command syntax (command syntax knowledge). Prompt 3 explains concepts of redirection in an abstract fashion, independently of syntax (conceptual 1/O redirection knowledge). Prompt 4 identifies the actual LO redirection symbols (I/O redirection syntax knowledge) required for the problem. Prompts 5 and 7 remind the user of the complete set of items that have already been identified in previous prompts. We assume that the latter prompts ease memory load for items. Prompt 6 is the first prompt that determines the order of commands and symbols for the user (providing command redirection knowledge and help in tracking intermediate results). This information is repeated in Prompt 8 . Finally, Prompt 9 gives the user the correct production.

The prompts were displayed in order of severity of the proposed cause of error; for example, the prompt telling the exact command names was always presented earlier than the prompt explaining the 
Table 2

Example of Task Description and Prompts for the Problem "nroff -ms ATT2 >ATT1"

\section{Iask Description}

Format the text in ATT2 using the -ms macro package and store the formatted version in ATT1

\section{Prompts}

Prompt 1. You will need to use the following command

One that will format the contents of a file using the -ms macro package

\begin{tabular}{l}
$\begin{array}{l}\text { Prompt } 2 \\
\text { command }\end{array}$ \\
nou will need to use this \\
of a file using the $\quad$-ms macro package \\
\hline $\begin{array}{l}\text { Prompt } 3 \quad \text { You will need to use a } \\
\text { special symbol that redirects command } \\
\text { output to a file }\end{array}$
\end{tabular}

Prompl 4 You will need to use the

arrow symbol " $z^{n}$ that redirects output

from a command to a file

Prompt 5 You will need to use the
arrow symbol " $z$ " and the command
nroff -ms

Prompt 6 You'll need to use an

nroff-ms on ATT2 (which will output

the formatted contents of ATT2), and

you'll need to redirect this output as

input to ATT1

Prompt 7 You will need to use
exactly the following command
elements (though not necessarily
in this order):
$\geq$ nroff-ms

$$
\begin{aligned}
& \text { Prompt } 8 \text { You'll need to use the } \\
& \text { command nroff -ms followed by the } \\
& \text { arrow symbol " } z^{\prime \prime}
\end{aligned}
$$

Prompt 9 The correct production is
nroff-ms ATI2>ATI1

Please enter this production now concept of redirection. We based this ordering on the Doane, Pellegrino, and Klatzky (1990) data, which showed that users acquire command names before they acquire redirection abilities. The ordering was also based on the model. Recall that in order to accomplish the redirection tasks, the model required knowledge that was more abstract, and according to the literature, this is more difficult for novices to acquire (e.g., Chi et al., 1981). Although the problems varied in the number of prompts that accompanied them, depending on the length of the target command sequence, all prompt series followed the same general structure and are categorized into nine ordered prompts.

Finally, we used physical $3 \times 5$ in. error cards that described the type of error made by the subject on an incorrect attempt. The three types were "Illegal," "Different: Legal but does something other than what the problem asks for," and "Keystrokes: Legal and does what the problem asks for but uses more than the minimum possible keystrokes." (The latter error category was intended to force subjects to use composite commands rather than a series of commands with temporary files in order to be correct.)

It is important to clarify the impact of presenting the prompts in a fixed order on the later interpretation of results. One question that we can ask is whether subjects need only receive concrete information about commands in order to produce them correctly, or whether they also require some ordering information. This experiment addressed this question. Another question is how much prompting a subject will have to receive before he/she can improve on a subsequent attempt or actually produce the correct composite command. This experiment addressed this question as well. There is also 
Table 3

Production Answers for Complete Problem Set

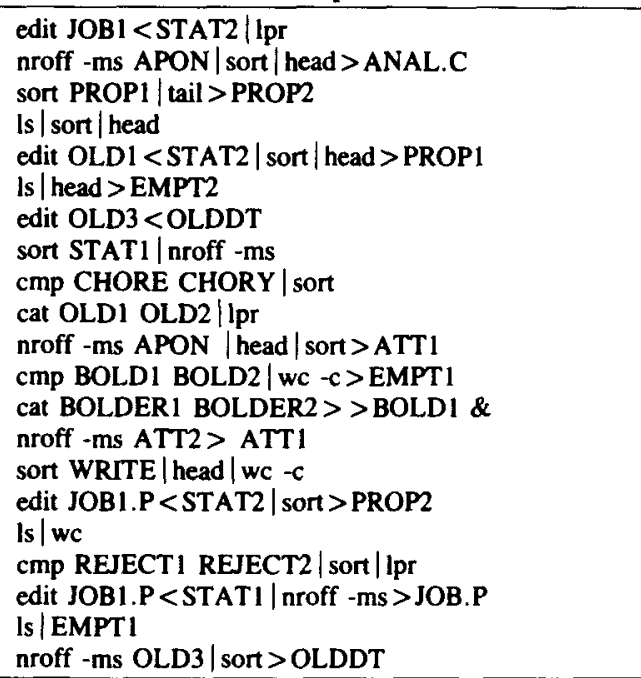

the question of whether certain permutations of prompt presentations would lead to different results because of additive or interactive effects among prompts. This experiment did not address this issue.

\section{Procedure}

The subjects were run individually for a single $2-h$ session. The experiment was made up of three sections: a questionnaire, an orientation, and the completion of the actual UNIX problems.

Orientation. To ensure that the subjects were familiar with the Macintosh II and the mouse, we had them read instructions for the experiment on the screen and use the mouse to click on button images in order to proceed from one page of instructions to the next. To familiarize the subjects with the specific procedures used in the composite command problems, the program presented them with two practice problems. The first practice problem was a difficult trivia question intended to require that subjects understood the function of, and procedures for, the help prompts. The second problem was a basic composite command problem intended to ensure familiarity with the style of problem presentation as well as some further familiarity with the help prompts.

Composite command problems. The subjects were given a series of 21 composite command problems. In this activity, the subjects were presented with a task statement on the computer screen, like that shown in Table 2, and they were asked to type a command or a series of commands that would accomplish the task. For each composite problem, the task statement appeared first, along with the directory listing of all file names used in the experiment. The subjects would then type their first attempt at a command or command series to accomplish the task. After the subject clicked the button image labeled "DONE," the program would evaluate the subject's attempt and give feedback of "Correct" or "Try again." If the subject's answer was not correct, the experimenter evaluated the subject's attempt and handed the subject the appropriate physical error card. After reading the error card, the subject clicked another button image; the program then erased the subject's attempt and revealed the first help prompt.

Once the help prompt was revealed, the subject tried again to give a command sequence that would accomplish the task. If the sequence was incorrect, the subject received another physical error card and was shown another help prompt. Throughout a given task, the task statement, directory listing, and all previous prompts remained visible; only the subject's previous attempts and physical error cards were removed. If at any time the subjects felt com- pletely stymied, they were able to type "no help." This indicated that the prompt did not provide them with any information or help that would allow them to change their production from their last attempt. They entered this rather than a solution attempt, in order to get the next help prompt. The procedure of subject attempt, error card, and help prompt continued until the subject typed the correct response. When the subject typed the required command sequence, the program displayed a "CORRECT!" screen and then revealed instructions for the next composite task.

\section{RESULTS AND DISCUSSION}

\section{Scoring Correct Command Productions}

Productions were scored as correct by the Macintosh computer (using a SuperCard program) if they matched the syntax of the idealized command (spaces were not parsed). Thus, a subject had to produce the command that required the least number of keystrokes (i.e., subjects could not substitute "sort file1 > temp; head temp > file2" for the command "sort file1 $\mid$ head $>$ file2").

\section{Partitioning Productions into Groups}

The data were analyzed by grouping all of the problems together, which allowed a comparison of all relevant effects of expertise level and prompt. However, this approach would have ignored the existence of an important learning curve. The learning curve is influenced by two aspects of the problems: their serial position, and the percentage of new knowledge required for solution. A fact in a problem was considered new knowledge if it had not been used previously by the subject in the experiment. In a multiple partial correlation, the two variables (i.e., serial position and percent new knowledge) were used to predict the number of prompts required to obtain correct production performance. Serial position was not a significant unique predictor of percent correct performance $(r=.008)$, whereas percent new knowledge was $(r=$ .493). Consequently, we will examine performance as a function of new knowledge. We split the problems into three groups according to an arbitrary but sensible criterion, those requiring $0 \%$ new knowledge ( 8 problems), those requiring $1 \%-59 \%$ new knowledge ( 10 problems), and those requiring $60 \%-100 \%$ new knowledge ( 3 problems). The number of problems entered in each knowledge group were identical for the three presentation orders that were used in this experiment. ${ }^{3}$

\section{Correct Productions as a Function of Prompt}

Figures $1 \mathrm{a}-1 \mathrm{c}$ show the cumulative percentage of correct composite productions for the three expertise groups as a function of prompt. The figure shows data for each of the percent new knowledge problem groups. Figure la shows data for the $60 \%-100 \%$ new knowledge group of problems, Figure $1 \mathrm{~b}$ shows the data for the $1 \%-59 \%$ new knowledge problems, and Figure 1c shows the same for the $0 \%$ new knowledge problems. The data are cumulative; subjects who correctly produced a problem at Prompt 4 were included as correct data points at Prompts 5-9 as well. Thus, at Prompt 9, all of the subjects in each expertise group were at $100 \%$ correct performance. ${ }^{4}$ As expected, 


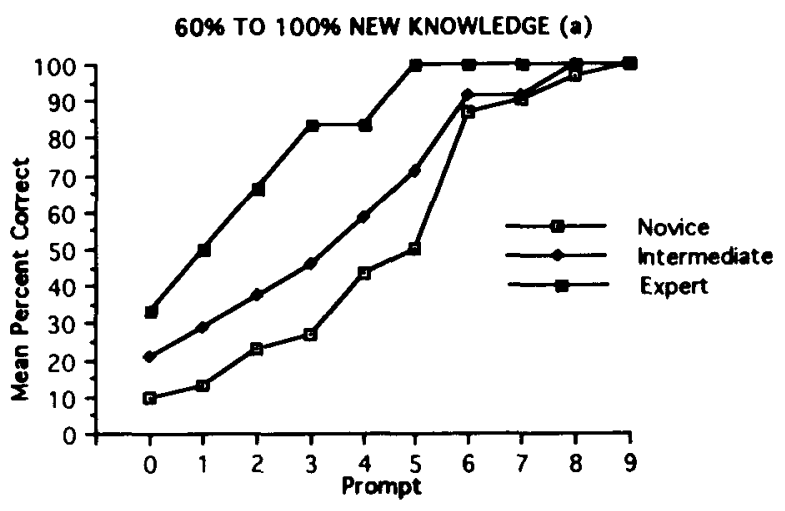

1\% TO 59\% NEW KNOWLEDGE (b)
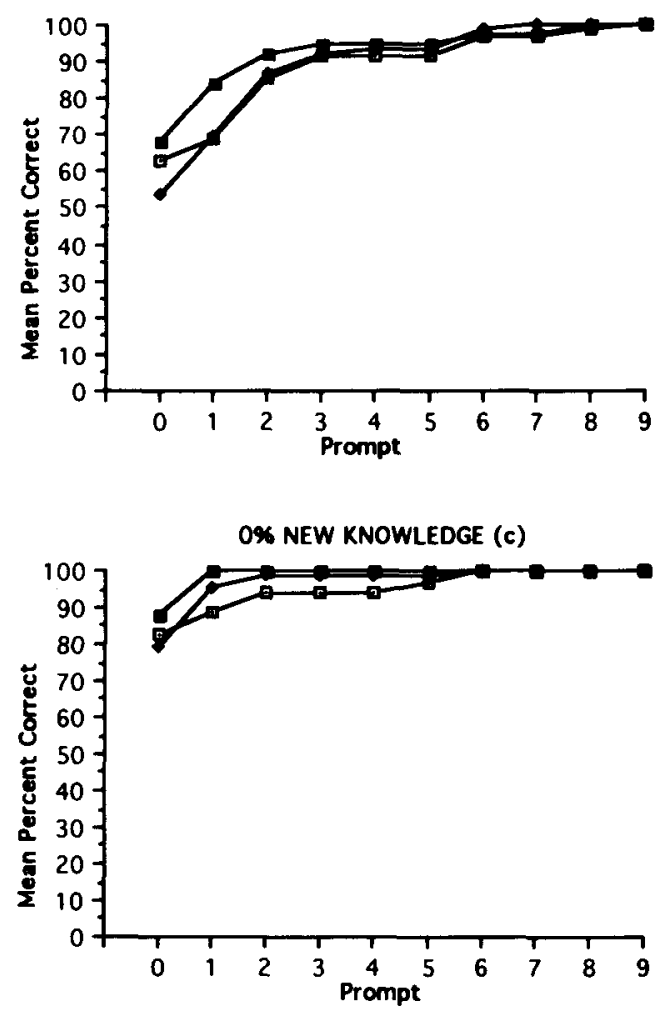

Figures la-1c. Mean percent correct productions for novice, intermediate, and expert groups as a function of prompt for $60 \%-100 \%, 1 \%-59 \%$, and $0 \%$ new knowledge problems.

across all three graphs, experts have the highest correct percentage overall, followed by the less expert groups. Focusing on the $60 \%-100 \%$ new knowledge problems (Figure 1a), we note that prompts have differential influences on correcting performance for the three expertise groups. For example, the change in percent correct performance from Prompt 3 to Prompt 4 is very small for experts, leading to the inference that Prompt 4 , which gives I/O syntax information (see Table 2) provided little or no new information to them. Conversely, the same change between Prompts 3 and 4 for the less expert groups is relatively large, suggesting that this prompt does provide them with some new information. These effects are attenuated for problems that require less new knowledge (Figures $\mathrm{lb}$ and $\mathrm{lc}$ ). This learning curve is apparently due to the tutoring value of the prompts and to practice on previous problems. It is interesting to note that for $0 \%$ new knowledge problems (Figure Ic), the novices and intermediates still had some problems, even though they had previously been exposed to all of the facts that they needed in order to successfully complete these problems. Experts only require a parsing of the problem at Prompt 1 in order to obtain perfect performance. Sometimes the experts realized that they had entered a typographical error, and the information that their command was incorrect was sufficient for them to correct the problem. Most of the time, however, the experts had dropped a command or forgotten part of the instruction, and the parsing prompt helped them realize that they had made this error. Novices, in contrast, only achieve perfect performance once they are exposed to the ordering information in Prompt 6. To analyze this effect, we will study the four types of component knowledge demonstrated in each attempt in detail.

\section{Scoring of Knowledge}

Each of the problems given to the subjects required a certain amount of the four types of component knowledge discussed earlier in the section on the UNICOM model. For example, the problem described in Table 2 requires two command syntax facts: "nroff -ms" as a command name, and that "nroff" takes a file argument. It requires knowledge of one $1 / O$ redirection syntax fact - that " $>$ " redirects output from a command to a file. The conceptual $\mathrm{I} / \mathrm{O}$ knowledge required is that redirection of input and output can occur, and that $\mathrm{I} / \mathrm{O}$ redirection can occur from commands to files. The required command redirection knowledge includes the fact that "nroff" output can be redirected to a file. Answers for all tasks were scored for the percentage of each type of knowledge displayed by a subject at each prompt level. Two raters worked together to score the productions and to develop a consistent scoring scheme that was relatively straightforward. For example, if a command requires two command syntax facts, and the attempt at Prompt 0 (before any prompts) shows evidence of only one of these facts, then the subject is credited for having $50 \%$ of the required command syntax knowledge for that task. As a specific example, for the command "ls > file," there are two command syntax facts ("ls" command lists file names of a directory, "Is" assumes a default directory), one I/O syntax fact ("> " redirects command output to a file), two conceptual I/O facts (I/O can be redirected, output can be redirected from a command to a file), and one conceptual command redirection fact ("ls" output can be redirected to a file). The production attempt of "ls" for this problem would be scored as having $100 \%$ of the command syntax knowledge and $0 \%$ of the other three types 


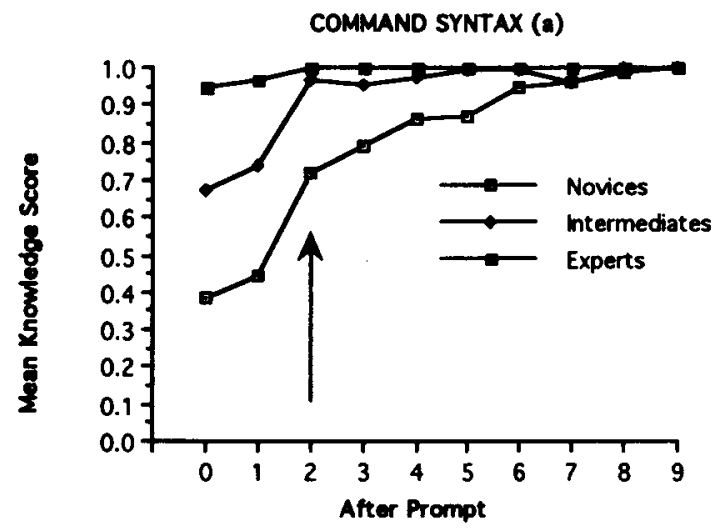

INPUT/OUTPUT SYNTAX (c)

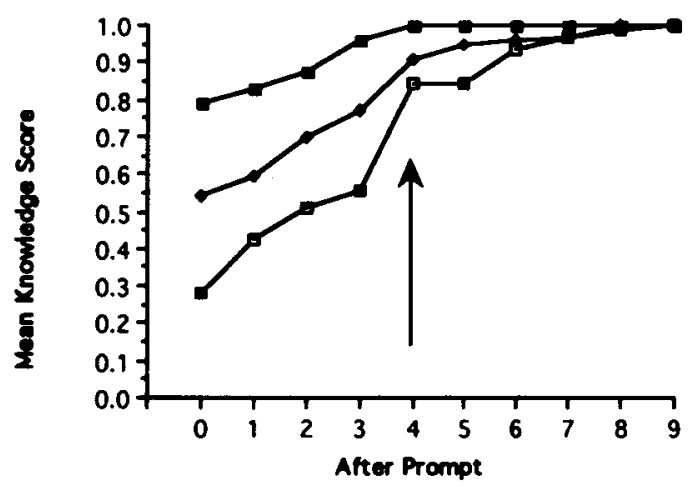

INPUT/OUTPUT REDIRECTION CONCEPTUAL (b)

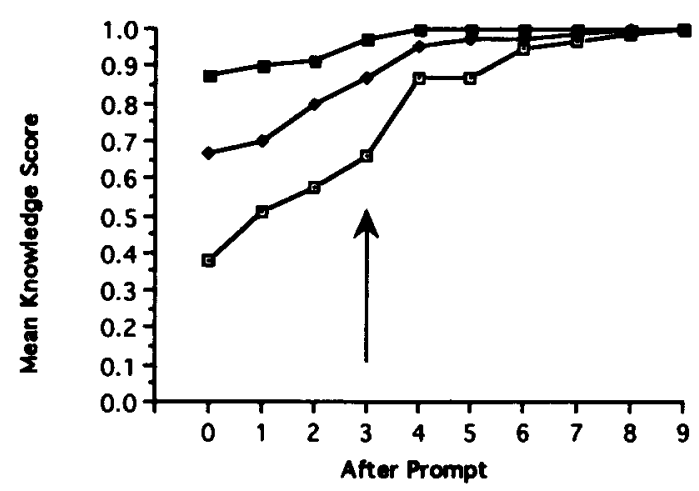

COMMAND REDIRECTION CONCEPTUAL (d)

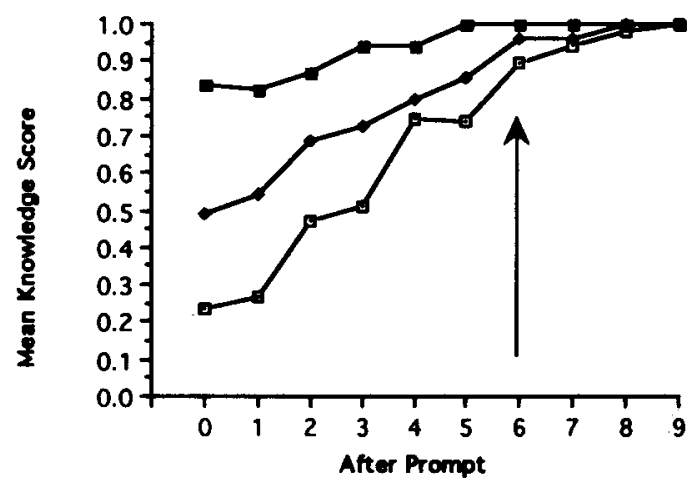

Figures 2a-2d. Mean command syntax knowledge, yO conceptual knowledge, L/O syntax knowledge, and command redirection knowledge scores for expert, intermediate, and novice groups for $60 \%-100 \%$ new knowledge problems as a function of prompt.

of knowledge. When a production was correct, all knowledge types were at $100 \%$ levels.

\section{Knowledge Analyses}

The $60 \%-100 \%$ new knowledge problems again provide the most interesting data. The other two problem groups show similar, but attenuated, expertise effects, and thus they will not be shown. Figures $2 \mathrm{a}-2 \mathrm{~d}$ show the mean knowledge scores for the three expertise groups after Prompts 0-9, for the $60 \%-100 \%$ new knowledge problems. The arrow markers specify which prompt first provided information relevant to the knowledge type displayed in the graph. For example, in Figure 2a, Prompt 2 is the first prompt that describes all of the command syntax knowledge required to complete the task (see Table 2 for an example of all prompt types described in this section), and the arrow marks knowledge displayed after presentation of Prompt 2. The component knowledge shown is higher than the percent correct scores shown in Figure la. This is because a subject attempt can show high, but not perfect component knowledge, and component knowledge must be perfect for an attempt to be entered as correct in Figure 1a.

Expertise and prompt effects. To determine whether amount of relevant knowledge is influenced by expertise and prompt, these data were subjected to a $3 \times 3 \times 4 \times 10$ analysis of variance (ANOVA), with expertise level (expert, intermediate, novice) as a between-subject variable, and percentage new knowledge, knowledge type, and prompt as within-subject variables. (In all cases, Greenhouse-Geisser degrees of freedom are used for within-subject variables [see, e.g., Winer, 1971]. This results in adjusted degrees of freedom for within-subject variables as a function of heterogeneity of variance.) This analysis resulted in a main effect of expertise level $\left[F(2,19)=4.91, M S_{\mathrm{e}}=.29, p<.04\right]$. Experts yielded significantly higher knowledge scores than did the other two groups across all three percentage new knowledge problem groups. There was also a main effect of knowledge type $\left[F(3,57)=20.55, M S_{\mathrm{e}}=.009, p<.01\right]$, with command redirection conceptual knowledge showing the lowest scores overall, as evidenced by a planned comparison $\left[F(1,19)=40.45, M S_{\mathrm{e}}=.466, p<.01\right]$. We did not obtain a knowledge type $\times$ expertise level interaction $(F<2)$, seemingly because of the expert-group ceiling effect.

To determine the specific effects of expertise, these data were further analyzed with planned comparisons. Novices and intermediates achieved significantly different knowledge scores than did experts $\left[F(1,19)=5.83, M S_{\mathrm{e}}=\right.$ 
$.295, p<.03]$. The experts possessed unique amounts of knowledge across all of the tasks. Novices and intermediates did not show significantly different knowledge scores across problems $\left[F(1,19)=3.53, M S_{\mathrm{e}}=.295\right.$, $p<.07]$.

There was a main effect of prompt $[F(9,171)=45.96$, $\left.M S_{\mathrm{c}}=.02, p<.01\right]$, as well as a prompt $\times$ expertise level interaction $\left[F(18,171)=5.42, M S_{e}=.02, p<\right.$ .01]. Performance improved as users received more prompts, though the amount of improvement was different for experts than it was for novices and intermediates. Experts appear to have reached ceiling performance more quickly than novices did.

Prompts influenced the four types of knowledge differently, as evidenced by a knowledge $\times$ prompt interaction $\left[F(27,513)=8.60, M S_{\mathrm{e}}=.002, p<.01\right]$. The influence of prompts on the four knowledge types was not equivalent for experts and the less expert groups $[F(3,54)$ $\left.=7.35, M S_{\mathrm{e}}=.022, p<.01\right]$. This suggests that our prompts influence the knowledge displayed in production attempts, and that the prompts differentially influence different types of requisite knowledge as a function of subject expertise. This important finding will be discussed further below.

Percentage new knowledge effects. As expected, the percentage of new knowledge that a problem required influenced knowledge scores $\left[F(2,38)=30.32, M S_{\mathrm{e}}=.13\right.$, $p<.01]$, and this effect was different for groups with varying levels of expertise $\left[F(4,38)=6.23, M S_{\mathrm{e}}=.13\right.$, $p<.01$ ]. Experts' performance changed less as a function of percentage of new knowledge than did the performance of less expert groups. The type of knowledge that a problem requires (i.e., command syntax, I/O syntax, L/O conceptual, command redirection conceptual) may be more or less difficult to use. The difficulty of using different types of knowledge seems to be a function of how much new knowledge a problem requires, as is evidenced by a knowledge type $\times$ percent new knowledge interaction $\left[F(6,114)=11.10, M S_{e}=.008, p<.01\right]$. Furthermore, prompts had a differential effect on performance as a function of the percentage of new knowledge that a problem required $\left[F(18,342)=28.86, M S_{\mathrm{e}}=.014, p<.01\right]$. Prompts were less influential on performance in $0 \%$ new knowledge problems as opposed to other problems, because all groups had learned from previous prompts.

The amount of new knowledge interacted with both the prompts and expertise, as is evidenced by a three-way interaction $\left[F(36,342)=4.91, M S_{e}=.014, p<.01\right]$, and with the prompts and the type of component knowledge required $\left[F(54,1,026)=4.78, M S_{\mathrm{c}}=.002, p<\right.$ $.01]$. It appears that the experts were less influenced by the percentage of new knowledge required, perhaps because overall this knowledge was less new to them than it was to the less expert groups. In a sense, they were not being tutored in this experiment to the extent that the less expert subjects were.

Retationship between percent correct and knowledge scores. It is important to realize that the knowledge scores are an indication of the levels of the four different types of knowledge described above that are displayed in an attempt. They are not necessarily an indication of a correct production. For example, a subject can show an increase in knowledge scores in Figures $2 \mathrm{a}-2 \mathrm{~d}$ and still not show an increase in percent correct performance in Figure 1. This occurred when a prompt had provided a subject with some information that enabled an improvement in performance yet the resulting production was still incorrect.

The difference between percent correct performance and the amount of knowledge displayed in an attempt can be examined by comparing the knowledge scores shown in Figures 2a-2d with the percent correct performance shown in Figure 1. Recall that in Figures 2a-2d, the arrow markers indicate the knowledge scores obtained after presentation of a prompt that provided the relevant information. For example, the arrow in Figure 2a points to command syntax knowledge scores obtained after presentation of Prompt 2, which helped with command syntax (see Table 2 for an example). Figure 2a suggests that, for all groups, the presentation of Prompt 2 improved command syntax knowledge and percent correct performance (see Figure 1a). However, the change in percent correct performance was less for the novice and intermediate groups (see Figure 1a). Figure 2a suggests that for less expert groups, the prompt was not sufficient to guarantee that subsequent attempts would show perfect command syntax knowledge. In contrast, experts only required one prompt detailing syntax to be able to display perfect command syntax knowledge.

Figure $2 \mathrm{~b}$ shows groups with improved $\mathrm{V} / \mathrm{O}$ conceptual knowledge scores for their attempts following the presentation of Prompt 3, which gave this information. Again, note that while the information helped all groups improve their $\mathrm{I} / \mathrm{O}$ redirection knowledge scores, the experts were best able to utilize this information to change their percent correct performance (Figure 1a).

Figure 2c shows that Prompt 4 appears to have assisted the novice group with $\mathrm{L} / \mathrm{O}$ syntax knowledge, and that the same trend holds to a lesser degree for the intermediates. Figure la suggests that this prompt increased the percent correct performance for the less expert groups. The experts, however, found this information useless.

Figures $2 \mathrm{~d}$ and la show similar trends in the change in command redirection knowledge for less expert groups following Prompt 6 , though certainly the expert data were influenced by a ceiling effect. This prompt gives both command redirection information and ordering information.

A summary of the trends found in correct performance as a function of prompt is also apparent in Figure 3. This figure shows the percent change in correct performance for the 60\%-100\% new knowledge problems for the first prompts that provided parsing of the instruction (Prompt 1), command syntax knowledge (Prompt 2), conceptual V/O knowledge (Prompt 3), 1/O syntax (Prompt 4), a first reminder of the items required for the production (Prompt 5), and the first command redirection and ordering information (Prompt 6). Overall, the experts and novices show very different changes in performance as a function of 


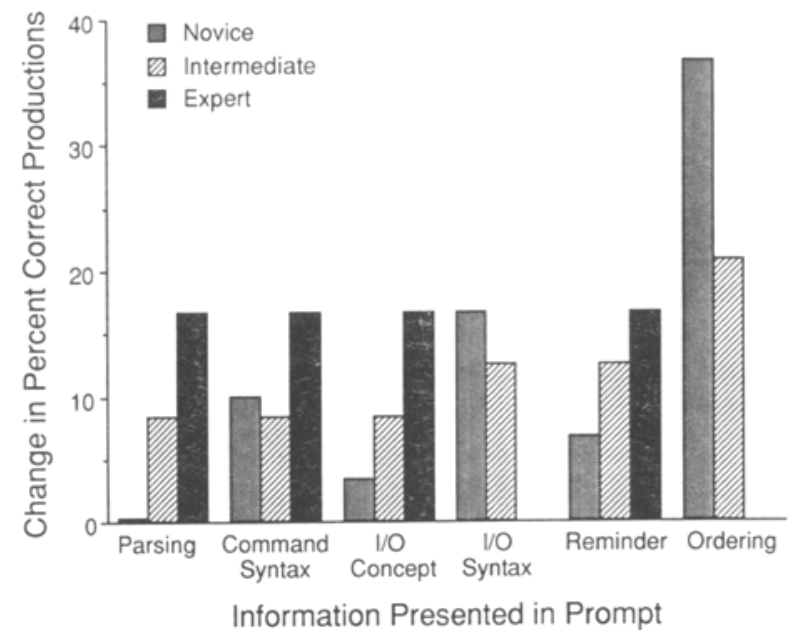

Figure 3. Changes in percent correct scores as a function of the type of information presented in a prompt for expert, intermediate, and novice groups for the $60 \%-100 \%$ new knowledge problems.

exposure to different types of prompts, and intermediates fall somewhere between the other two groups.

Summary of percent correct and knowledge score analyses. It appears that novices and intermediates need help with I/O syntax, but that this is not a problem for the experts. Experts get help from an abstract statement of $1 / 0$ conceptual knowledge (see Table 2 for an example), while this statement is less worthwhile to the novice and intermediate groups. This finding is in line with the expertise literature (e.g., Chi et al., 1981), which suggests that experts can process more abstract information than novices can. Certainly Prompt 3 is more abstract in the sense of providing less specific information than do Prompts 2 and 4.

Experts also successfully utilize a reminder prompt that lists all of the command and $\mathrm{I} / \mathrm{O}$ elements together in one prompt (Prompt 5). Experts who have not solved a problem correctly by this point have often represented the problem with one command reversed or dropped; this prompt reminds them that they have forgotten an element, and they can use this information to make a correct production. The less expert subjects seem to have more problems at this stage than just knowing the elements, so while they receive help from Prompt 5, they cannot achieve perfect production performance if given just a list of the items. Their response to the ordering prompts (see Prompts 6 and 8 in Figure 1a) makes this clear. These are the first prompts that tell users what order they must use for the commands-for example, that "nroff -ms" must first be performed on ATT2, that this output must then be redirected to the file ATT1, and so on. Although the experts have solved all ordering problems before this information is presented, this ordering information helps the less expert groups quite a bit.

\section{Scoring Production Errors}

So far, we have provided evidence suggesting that the less expert groups need assistance with ordering. We shall now consider evidence that the less expert subjects need this information because they lack the requisite conceptual redirection knowledge or have a working memory load problem, or both. ${ }^{5}$ One possible reason that less expert subjects fail to produce composites prior to reading the ordering prompt is that they lack the requisite command-specific redirection knowledge. In this case, the ordering prompt helps less expert users, because it is the first prompt that provides them with this knowledge.

Another possible reason that less expert subjects are helped by the ordering prompt is that it aids users who have memory load problems. It may be that keeping track of the order of commands and their intermediate results taxes working memory, and in this case, that the ordering prompt helps because it provides a memory aid. The work of Murdock (1974) and Anderson and Jeffries (1985) suggests ways to obtain evidence for such problems from these data. Murdock argued that memory losses are evidenced in both item and order errors, and that item errors indicate more serious losses of memory. His work shows that the first memory lost is that of the order of item presentation, and that this is followed by a complete loss of memory for an item. Anderson and Jeffries also suggest that items can be systematically deleted from, in their case, LISP programming code, as a result of working memory limitations.

For the present study, the presence of each type of error is indicative of either a working memory load problem or a lack of knowledge, or both. That is, users may delete components of a command because they do not know the components until they are prompted with the information (indicating a knowledge deficit). They may also delete items from an attempt because they have trouble keeping all elements of a command in memory even after they are prompted with the information (memory load problem). Finally, there may be an interaction between the knowledge prerequisites and memory demands that together lead to poor performance (see, e.g., Chase \& Ericsson, 1982).

We scored user attempts for the number of deletions and substitutions of command elements to obtain a measure of erroneous performance. For deletions, we counted the number of items deleted in each command attempt. The items included filenames, utilities (e.g., "ls," "sort"), and specialty symbols (e.g., ">," ""p). To determine substitution errors, we counted the number of substitutions for utility, file, and specialty symbols. Errors that appeared to be order errors (e.g., "sort| head" for "head|sort") and attempts that included true command substitutions (e.g., "tail | sort" for "head | sort") were counted as substitutions. ${ }^{6}$ We also measured the addition of symbols (e.g., "sort| head| lpr" for "sort| head"), but there was an insufficient number of these errors or other types of errors to warrant analysis or discussion.

\section{Error Analyses}

Deletion and substitution errors. Figures $4 a-4 b$ show the mean deletion and substitution errors for the three groups in the $60 \%-100 \%$ new knowledge problems as a 

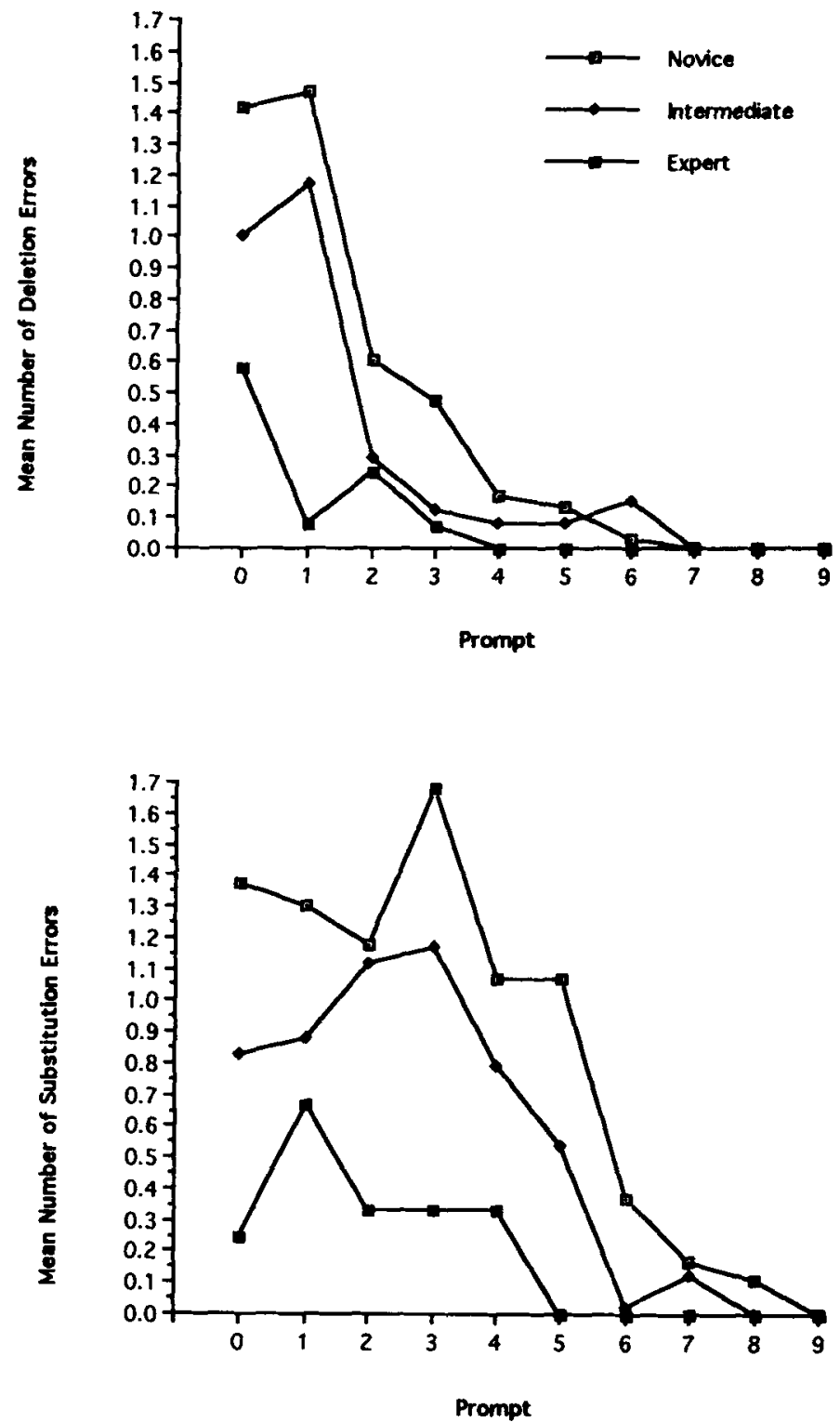

Figures $4 \mathrm{a}-4 \mathrm{~b}$. Mean deletion (top panel) and substitution (bottom panel) scores for expert, intermediate, and novice groups as a function of prompt for $60 \%-100 \%$ new knowledge problems.

function of prompt. The graphs for the other two new knowledge classifications show similar trends that are attenuated by the learning curve and thus are not presented. Novices and intermediates show the greatest number of deletion and substitution errors, suggestive of either working memory load or knowledge deficit problems. To determine whether the number of deletions and substitutions is influenced by expertise and prompt, these data were subjected to separate $3 \times 3 \times 10$ ANOVAs with group (expert, intermediate, novice) as a between-subject variable, and new knowledge and prompt as within-subject variables. These data show significant effects of expertise level [deletion, $F(2,19)=4.24, M S_{\mathrm{e}}=.204, p<.03$; substitution, $\left.F(2,19)=6.23, M S_{\mathrm{e}}=.427, p<.01\right]$. Nov- ices committed the greatest number of errors, relative to the expert and intermediate groups, as supported by planned comparisons [deletion, $F(1,19)=8.04, M S_{\mathrm{e}}=$ $.02, p<.01$; substitution, $F(1,19)=11.04, M S_{\mathrm{c}}=.043$, $p<.02$ ]. There was also a main effect of prompt [deletion, $F(9,171)=15.68, M S_{\mathrm{e}}=.094, p<.01 ;$ substitution, $\left.F(9,171)=17.61, M S_{\mathrm{c}}=.104, p<.01\right]$. The deletion errors decreased radically for all groups after the first command syntax prompt (Prompt 2), and substitution errors decreased after the first ordering prompt. It is difficult to explain the rise in substitution errors for the less expert groups following Prompt 3. Recall that this prompt presented the abstract information about $1 / O$ concepts (see Table 2). It is possible that this general state- 
ment confused the less expert groups and that they tried new commands and orderings in response. Also note the lack of a drop in substitution errors by the novices at Prompt 5, consistent with our earlier hypothesis that they would obtain little help from the listing of elements in a command. If this had been the case, Prompt 5 would have had an impact on their deletion and substitution errors and on their percent correct performance. Instead, they required specific ordering information in order to either decrease their memory load errors or increase their knowledge and improve their correct performance. Finally, the percentage of new knowledge required influenced the number of errors [deletion, $F(2,38)=13.17, M S_{\mathrm{e}}=$ $.293, p<.01$; substitution, $F(2,38)=38.09, M S_{\mathrm{e}}=$ $.35, p<.01]$. Errors were highest for the $60 \%-100 \%$ new knowledge problems. For substitutions, the effect of new knowledge was stronger for the less expert groups than for the experts $\left[F(1,23)=9.07, M S_{e}=.035, p<.01\right]$.

Effects of command length. In addition, if we perform a correlation analysis to determine the effect of length of command (where we assume that a longer command in terms of number of commands, symbols, and filenames both taxes memory load and requires more knowledge than do shorter commands) on number of deletion and substitution errors, we find that novice performance shows a significant correlation between length of command and the most severe type of error, deletions $[r(207)=.25$, $p<.01]$, and also with substitutions $[r(207)=.21, p<$ .01]. Intermediates show a weaker but significant correlation between command length and deletions $[r(165)=$ $.17, p<.03$ ], and a significant correlation between length of command and substitution errors $[r(165)=.30$, $p<.01]$. Experts show no significant increase in their deletion or substitution errors as a function of command length. Since an increase in command length taxes working memory, and deletion and substitution errors are indicative of working memory load, these results are consistent with the hypothesis that memory processes play a significant role in composite performance.

Effects of redirection symbol combinations. Knowledge also plays a role, however. We can examine the influence of knowledge by differentially rating composites for the knowledge required for their production. Composites that require the use of different types of redirection symbols (e.g., both a pipe and a filter in one command) can be termed mixed composites, and these require significantly more pieces of knowledge about redirection properties of symbols and of commands than do composites that are not mixed (e.g., commands requiring two pipes). If we correlate the mixed rating of a command with the incidence of deletion and substitution errors for each group, partialing out the effect of command length described above, we find that the knowledge required in mixed commands is a significant problem for less expert groups. The results of this analysis are shown in Figure 5. Basically, experts are the only group that does not show a significant relationship between mixed command type and deletion and substitution errors. This suggests that they alone have both the knowledge and the memory

\begin{tabular}{|c|c|c|c|}
\hline Group & Predictor & Deletions & Substitutions \\
\hline Novice & $\begin{array}{l}\text { Length of } \\
\text { Command } \\
\text { Mixed Type }\end{array}$ & $\begin{array}{l}r(207)=.07 \\
r(207)=.158\end{array}$ & $\begin{array}{l}r(207)=.044 \\
r(207)=.154 \text { * }\end{array}$ \\
\hline Intermediate & $\begin{array}{l}\text { Length of } \\
\text { Command } \\
\text { Mixed Type }\end{array}$ & $\begin{array}{l}r(165)=.005 \\
r(165)=.192\end{array}$ & $\begin{array}{l}r(165)=.143^{* 4} \\
r(165)=.122\end{array}$ \\
\hline Expert & $\begin{array}{l}\text { Length of } \\
\text { Command } \\
\text { Mixed Type }\end{array}$ & $\begin{array}{l}r(81)=.055 \\
r(81)=.13\end{array}$ & $\begin{array}{l}r(81)=.009 \\
r(81)=.125\end{array}$ \\
\hline
\end{tabular}

Figure 5. Partial correlations between command length, mixed type of composite, and deletion and substitution errors for novice, intermediate, and expert groups. $\quad{ }^{*} p<.05$ or better. $\quad * 0<.06$.

strategies (e.g., chunking) that are required in order to deal with the composite commands.

Summary of error analyses. Thus, the hypotheses suggested by our knowledge analysis were supported. There appear to be separate components of knowledge above and beyond the simple command knowledge that is required in order to produce composites. There is evidence that the less expert groups made more errors that were consistent with a memory load hypothesis than did the experts. And the extent of these errors decreased radically for less expert groups when they were helped with ordering information. However, retrieving the elements is only part of the problem in constructing a composite command. We can do knowledge-rich tasks if the environment provides us with cues to activate the relevant knowledge (e.g., prompts). However, knowledge interacts with the environment and with memory processes. To chain elements together to make a composite requires relating the pieces of knowledge in an ordered fashion and tracking the intermediate results. And this task makes significant demands on memory. The fact that UNIX gives no information regarding intervening results interacts with the user's need for keeping track of intervening results and produces both heavy knowledge demands and a memory load.

\section{GENERAL DISCUSSION}

Theoretical analyses can have value for the solution of problems encountered in the applied world, and problems encountered in the applied world can enhance the development of theory. Two major contributions of the present work are described below.

\section{Contribution of Theory to Real-World Problem Solving}

Understanding the problem. Why does it take 5 years to learn to effectively produce composite UNIX commands? Norman (1981) suggested that one of the problems for UNIX users results from the arcane names of single commands. The Doane, Pellegrino, and Klatzky (1990) data show that command names are just the tip of the iceberg. And our UNICOM modeling work suggests that the composition of composites is indeed a difficult action planning problem. Composite production requires 
knowledge of syntax for both command and redirection symbols. The production of composites also requires sophisticated conceptual knowledge about redirecting command input and output. Further, creating an action plan for producing composites poses large knowledge demands and a working memory problem because it requires ordering command elements together in a systematic fashion in the absence of any feedback about intermediate results (Doane, Kintsch, \& Polson, 1989, 1990). While our theoretical analysis suggested some answers, the answers required empirical support. This study provides much of that support.

Our finding that users vary markedly in their responses to very different classes of prompts as a function of expertise supports the UNICOM analyses. As we have stated, the prompts provided command syntax knowledge, I/O redirection syntax knowledge, command redirection conceptual knowledge, and I/O redirection conceptual knowledge, and they helped with memory load by listing all of the elements and by explicitly stating intermediate results. The results suggest that novices and intermediates lack both specific syntax knowledge and more general conceptual redirection knowledge. If prompted with specific syntax knowledge, novices and intermediates can increase their chances of producing a correct composite. However, novices and intermediates cannot utilize our more abstract prompt (Prompt 3), which provides the conceptual redirection information knowledge that they need (see Figure 2b) in order to increase their correct performance (see Figure la). Our experts, in contrast, can use this information effectively to increase their performance. This finding is consistent with the literature on expertise, which suggests that experts can utilize abstract information, whereas novices cannot (e.g., Chi et al., 1981).

Novices and intermediates need help in retrieving the elements that go into making a composite; but for many of them, this is not enough. They also need help in ordering the elements-or in constructing a command. When they receive a prompt that helps them order the items, their performance improves. There is evidence that ordering the elements challenges their knowledge and taxes their working memory. They delete and substitute many more components of a composite than do experts, and these errors decrease markedly once ordering information has been given. In contrast, experts attain almost perfect performance before any ordering information is given.

Thus, the hypotheses suggested by the UNICOM construction-integration model were supported. There appear to be separate components of knowledge above and beyond the simple command knowledge required for the production of composites. Furthermore, retrieving the elements is only part of the problem in composing a composite. To chain these elements together is another aspect of the problem, and this requires relating the pieces of knowledge in an ordered fashion and tracking the intermediate results.

These data suggest future studies that would more clearly separate the working memory load problems from the knowledge prerequisites. Recall that Prompt 6 in this study provided conceptual knowledge of redirection and helped users with ordering and keeping track of the intervening results. Future studies could present the conceptual command redirection knowledge in a separate prompt (e.g., "nroff output must be redirected to a file"). This could be followed by a prompt that gave only the ordering information and information about intervening results (it is not clear how to separate these two pieces of information in a prompt), and as such it would present no new knowledge and would only be providing help with working memory.

Suggesting design solutions. Can our theoretical analysis contribute to system design? It is interesting to note that none of the new graphical user interfaces for UNIX assist the user with composites. Although the theory does not suggest specific new system designs, it does suggest that new designs should aid users both with the conceptual knowledge required for the production of composites, and with keeping track of the intermediate results. One suggestion, then, is that there should be help prompts to assist users in composing composites-following the guidelines suggested by these data. For example, a general prompt will not help a novice.

Our analyses point out problems that need repair, but they do not show how the repair should be done. Nor do we propose that this is a comprehensive list of problems. The solutions that we discuss need not be graphical per se; we are commenting on potential graphical solutions because that is a new design form for UNIX user interfaces. Specifically, these analyses have led us to think that a graphical user interface that would clarify the redirection properties of commands might be beneficial to users. The model does not suggest a specific design. Rather, the resulting knowledge analyses suggest what aspects of a design will be important to a user, and this can be empirically evaluated. Furthermore, we are in a position to suggest that the visual qualities of a graphical user interface will not be the significant property; it will be the extent to which visualization makes transparent the redirection properties of commands and assists with ordering.

We are planning studies that will be done with a modified version of a graphical user interface for composites independently designed by Foley and one of his students (Jovanovic \& Foley, 1986) to test users on composites. In the interface, a user can enter a composite mode and have context-sensitive feedback about the intervening results. This type of feedback, in combination with a command space showing the command redirection properties of each command entered, should aid users with both knowledge demands and working memory load. Thus the theory has given us empirically supported insight into a real problem, and these insights are driving our research to make specific design modifications and evaluations.

Suggesting a methodology. What we have done in the context of a theory of comprehension is to provide a detailed knowledge analysis for UNIX users, and this has suggested possible design solutions that can be tested em- 
pirically. This methodology can be used to evaluate other complex system interface designs, and as such it is a methodological contribution to the applied research area.

\section{Real-World Problems Driving Theory Evolution}

So far, we have shown how we used the constructionintegration theory in our applied research, and how we have found support for our theoretical analyses. Presently we will describe the evolution of the theory thus far, as well as our plans for future theoretical development.

Current theory development. Applied research can drive a theory to expand, and we have extended the construction-integration theory as a result of this effort. First, we have extended the theory to another, more complex domain. The theory has previously been applied to text comprehension and to mathematical problem solving (Cummins, Kintsch, Ruesser, \& Reimer, 1988; Kintsch, Welsch, Schmalhofer, \& Zimmny, 1990), and now it has been applied to the production of novel, complex action planning in UNIX.

Second, to build the UNICOM construction-integration model, we had to extend the theory to include causal planning mechanisms that are found in traditional planning systems (see also Mannes \& Kintsch, 1991). For text comprehension data (e.g., Kintsch, 1988), the associative structure found in the initial conception of the constructionintegration theory is sufficient. For the UNIX work, the causal chaining between plan elements representing components of a composite is required.

Future theoretical work. The theory must be expanded further to remain fruitful in our current efforts to account for differences in knowledge as a function of expertise. Kintsch (e.g., 1988) has proposed that the overlap that exists between a network of propositions created by a text and the corresponding network of the reader determines what propositions will be remembered and processed. This notion can be extended in the UNIX work to suggest that the overlap between a network of propositions resulting from an incoming instruction or prompt and that of the user will dictate the user's recall of syntax and concepts relevant to the task, as well as dictate what the user will attend to. For example, a prompt may not overlap with novices' knowledge sufficiently to dictate their attention or processing of that material. This would explain why, for example, the abstract prompts are not of much use to the novices. This explanation is consistent with previous work on the construction-integration theory, but it must be studied further to be supported.

Direct support will be provided by extending our modeling work to account for the current prompt data. We are currently building models of individuals in this study, running simulations by giving UNICOM prompts, and evaluating prompt activation within the individual's network. We hope to determine whether the overlap between the knowledge in a prompt and an individual's network of knowledge dictates the usefulness of the prompt.

This work will have clear implications for computeraided instruction and for system design. For example, an intelligent help system could assess the existing network of knowledge possessed by a user of some type of discriminant analyses (i.e., some types of errors might do a good job of predicting what other knowledge a user has or lacks; see Doane, Pellegrino, \& Klatzky, 1990). The system could then calculate the overlap between, for example, one of three prompts that it could give to the user, and choose the one that had the highest overlap. If overlap effectively predicts a higher probability of processing and use of information, this would assist the user's skill acquisition tremendously. The system might also be able to observe the evolution of user knowledge with the acquisition of expertise and modify help information accordingly. This work would have clear implications for theories of expertise. If we could explain the attention to abstract versus concrete material in terms of the activation that a fact receives in a network of propositions, we might be able to model the transition from novice to expert in many different domains and explain the rich set of expert/novice differences that have been reported in the literature (e.g., Chi et al., 1981).

The theory will have to be extended in another fashion. Currently, the associative structure in the theory takes labeling very literally. For example, there are no natural links between commands that are very different on a syntactic level but that might be similar on a structural level. For example, the command "sort file|lpr," which prints the sorted contents of a file on the line printer, does not have much in common with the command "head file| wc," which counts the number of words in the first 10 lines of a file. However, structurally, these commands are similar in that they use a pipe in the middle and require that the output of the first command be redirected as input to the second command. Although linking these together on the basis of structural aspects is simple computationally, we would like the links to fall naturally out of the cognitive architecture as a function of user knowledge, and we would like the theory to predict what links of this type will exist. For example, experts may see this structural similarity because of their knowledge of piped commands, whereas novices may not.

In summary, we have made contributions to the theory by researching a complex applied problem, and to the applied problem our theoretical analyses have proposed solutions. We are optimistic about the continued contributions resulting from the interaction between this theory and our applied work in the future.

\section{REFERENCES}

Anderson, J. R. (1983). The architecture of cognition. Cambridge, MA: Harvard University Press.

ANDERSON, J. R., \& JEFFrIES, R. (1985). Novice LISP errors: Undetected losses of information from working memory. HumanComputer Interaction, 1, 133-161.

Card, S. K., Moran, T. P., \& Newell, A. (1983). The psychology of human-computer interaction. Hillsdale, NJ: Erlbaum.

ChI, M. T. H., Feltovich, P. J., \& Glaser, R. (1981). Categorization and representation of physics problems by experts and novices. Cognitive Science, 5, 121-152. 
Chase, W. G., \& Ericsson, K. A. (1982). Skill and working memory Psychology of Learning \& Motivation, 16, 1-58.

Cummins, D. D., Kintsch, W., Ruesser, K., \& Reimer, R. (1988) The role of understanding in solving word problems. Cognitive Psychology, 20, 405-438.

DonNe, S. M., Kintsch, W., Polson, P. (1989). Action planning: Producing UNIX commands. In Proceedings of the Eleventh Annual Conference of the Cognitive Science Society (pp. 458-465). Hillsdale, NJ: Erlbaum.

Doane, S. M., Kintsch, W., Polson, P. G. (1990). Understanding UNIX commands: What experts must know (Tech. Rep. No. 90 1). Boulder, CO: University of Colorado, Institute of Cognitive Science.

Doane, S. M., Pellegrino, J. W., \& Klatzky, R. L. (1990). Expertise in a computer operating system: Conceptualization and performance. Human-Computer Interaction, 5, 267-304.

Jovanovic, B., FoLEY, J. D. (1986). A simple graphics interface to UNIX (Tech. Rep. No. GWU-IIST-86-23). Washington, DC: George Washington University, Institute for Information Science and Technology.

KINTsCH, W. (1988). The use of knowledge in discourse processing: A construction-integration model. Psychological Review, 95, 163-182.

KintsCh, W., WelsCh, D., Schmalhofer, F., Zimmey, S. (1990). Sentence memory: A theoretical analysis. Journal of Memory \& Language, 29, 133-159.

LARKIN, J. H. (1983). The role of problem representation in physics. In D. Gentner \& A. Stevens (Eds.), Mental models (pp. 75-98). Hillsdale, NJ: Erlbaum.

Mannes, S. M., \& Kintsch, W. (1991). Routine computing tasks: Planning as understanding. Cognitive Science, 15, 305-342.

MURDOCK, B. B. (1974). Human memory: Theory and data. Hillsdale, NJ: Erlbaum.

Norman, D. A. (1981, November). The trouble with UNIX. Datamation, pp. 139-150.

NewelL, A. (1987). Unified theories of cognition (The 1987 William James Lectures). Cambridge, MA: Harvard University Press.

Polson, P. G., \& LEwIs, C. H. (1990). Theory-based design for easily learned interfaces. Human-Computer Interaction, 5, 191-220.

VAN DuJ, T. A., \& KINTSCH, W. (1983). Strategies of discourse comprehension. New York: Academic Press.

Wertheimer, M. (1982). Productive thinking. Chicago, IL: University of Chicago Press.

WINER, B. J. (1971). Statistical principles in experimental design (2nd ed.). New York: McGraw-Hill

\section{NOTES}

1. We are not suggesting that UNICOM is the only model that could have been used. We are pleased that we were able to use a model that has been used to explain other aspects of cognition, such as math problem solving and text comprehension (see, e.g., Kintsch, 1988), because this supports the goal of developing a unified theory of cognition (Newell, 1987).

2. One possible concern is that the novel problems might not make semantic sense to the subjects and that this would influence their performance. However, subjects did not indicate that they found the problems confusing or nonsensical. Furthermore, Doane, Pellegrino, and Klatzky (1990) provide comprehension data which suggest that the novel problems do make semantic sense to UNIX users.

3. The choice of a cutoff at $0 \%$ new knowledge is intuitive. The choice of $60 \%$ for a separation of the other two groups is based simply on the differences in knowledge required by the problems. There is a large gap in average new knowledge required for solving problems above the $60 \%$ cutoff value and for solving those below the $59 \%$ cutoff level.

4. A cumulative graph is used because a noncumulative graph shows confusing drops in percent correct performance. As a hypothetical example, assume that we ran an experiment with only one composite problem, that a majority of subjects in an expertise group solved the problem after Prompt 1, but that only 1 subject solved the problem after Prompt 2. A noncumulative graph would show a drop in percent correct performance between Prompt 2 and Prompt 3. In fact, the group has an overall increase in percent correct performance when the additional subject solves the problem, and this is represented by a cumulative graph.

5. We thank an anonymous reviewer and Susan Dumais for calling the knowledge argument to our attention.

6. True command substitutions account for very few substitution errors. This is particularly true after the presentation of Prompt 2, which explicitly provides subjects with command syntax knowledge. True command substitutions that follow the presentation of Prompt 2 are considered to be due to a memory load problem rather than to a knowledge deficit.

(Manuscript received April 17, 1991; revision accepted for publication March 8, 1992.) 